\title{
Understanding the Effects of Diet on Gut Microbiota in Obesity: A Review
}

\author{
Lima Hazarika ${ }^{1 *}$, Swaraj Saikia ${ }^{2}$ and Supriyo Sen ${ }^{1}$ \\ ${ }^{1}$ Department of Biosciences, School of Life Sciences, Assam Don Bosco University, \\ Sonapur, Assam, India \\ ${ }^{2}$ Institute of Sports Science and Technology (ISST), Pune, Maharashtra, India \\ *Corresponding Author: Lima Hazarika, Department of Biosciences, \\ School of Life Sciences, Assam Don Bosco University, Sonapur, Assam, India. \\ DOI: $10.31080 /$ ASNH.2020.04.0695
}

Received: March 10, 2020

Published: April 22, 2020

(C) All rights are reserved by Lima Hazarika. et al.

\begin{abstract}
Background: Obesity and associated disorders have reached an epidemic proportions worldwide. Studies have linked the condition to genetics as well as with lifestyle disorder. Microbial insights have helped in the understanding of gut microbiota contribution to many human health conditions.

Methodology: A literature search of electronic databases was performed, using the search syntax: Gut microbiota, diet, obesity, pathogenesis of obesity to build and summarize the broad view of the present paper.

Summary: The review discusses the pathogenesis of obesity, interactions of gut microbiota and diet in obesity and the effect of the dietary components intervention on the human health status. Reviewing the studies, it was evident that gut microbes have a significant role and can impact host metabolism via signalling pathways in the gut, with effects on inflammation, insulin resistance, and deposition of energy in fat stores. The host genetics, stress, age and gender contribute to the host-microbiota interactions in obesity. The macronutrients, micronutrients, minerals, fats, bioactive nutrients and food additives in diet interact with the gut microbiota to produce metabolites that modulate host metabolism in both beneficial and detrimental ways. The review attempted to evaluate the impact of dietary components on gut microbiota to disease onset, further identifying the research challenges and discuss the future consideration in clinical interventions that can be translated into clinical practice. Multidisciplinary research in this field will be helpful to provide therapeutic potential of gut microbes in metabolic disorders and obesity treatment.
\end{abstract}

Keywords: Nutrition; Weight; Microbiota; Gastrointestinal Tract (GIT); Host; Microbiome

\section{Introduction}

Overweight and obesity are significant health risk for various chronic diseases including diabetes, cardiovascular infections and cancer, is a serious health concern in developed countries but now, it has been growing in the urban settings of low- and middle-income countries [1]. The development of obesity directs towards many events like consumption of highly calorific food, lifestyle, less or lack of physical activity as well as genetic predisposition resulting in overweight and obese individuals in society.

Research studies indicate that diet plays a significant role in different chronic diseases like inflammatory bowel disease, obesity, type 2 diabetes, cardiovascular disease, and even cancer, thereby shaping the microbiome of the host [2]. The connection among diet, environmental and genetic factors making microbiota to affects the host immune and metabolic system result in disease risk and this has started to get targeted for therapeutic utility [3]. Obesity, reported as heritable trait has become alarming risk factor for type 2 diabetes, heart disease, and hypertension. The condition is linked to cardiovascular risk factors such as glucose intolerance and dyslipidemia and is associated with reproductive conditions like infertility and PCOS and metabolic disorders [4]. Environmental factors such as diet and nutrition, exercise and lifestyle are important but there is considerable evidence that genes also have a significant role in its pathogenesis. Genetic and internal environmental factors such as host microbiota and niches in the gastrointestinal tract also interact to regulate body weight [5]. WHO reported genetic variants of major candidate genes accounts an increase from $10 \%$ to about $13.1 \%$ prevalence of obesity among adults, globally $[5,6]$. This seem to be very complex entity altogether and would require a good understanding of multiple contributors viz., common genetic polymorphisms, diet and life style, host microbiota, related metabolic and other pathways while determining the therapeutic utility for overweight and obesity. This article reviewed the studies to understand the growing association of diet, microbiota and host that leads to obesity onset and metabolic diseases and discuss future considerations for multidisciplinary research.

\section{Pathophysiology of obesity}

Obesity is defined as abnormal or excessive fat accumulation that may be harmful to health [7]. Etiology of obesity has been 
associated with poor diet and insufficient physical exercise leading to energy imbalance between calories intake and calories expended, involving a complex interaction among life style, genetics, epigenetics, neuronal and hormonal mechanism, and the environment $[8,9]$. Emerging evidence suggests that dietary habits like high-sugar food and saturated fats, trans-fatty acids increases the risk of chronic vascular disease by elevating blood serum concentrations of total and LDL cholesterol, contributing to anxiety and mood disorders presenting a bi-directional associations with obesity [10]. With host genetics, factors like geographical location, stress across the life span, age and gender, excessive/inappropriate antibiotics or drug use, consequences of socioeconomic development like increased sedentariness or less physical activity has become a threat to the diversity of the gut microbiome (Figure 1). Lifestyle changes in societies, increased intake of preserved and processed food of higher calorie with lower nutritional value also contribute to onset of obesity. These factors altogether compromise the integrity of the gut microbiome through different physiological process and diversely impact on dynamic features of the gut ecosystem such as microniches, $\mathrm{pH}$ gradients and dynamic microbe-tissue interactions.

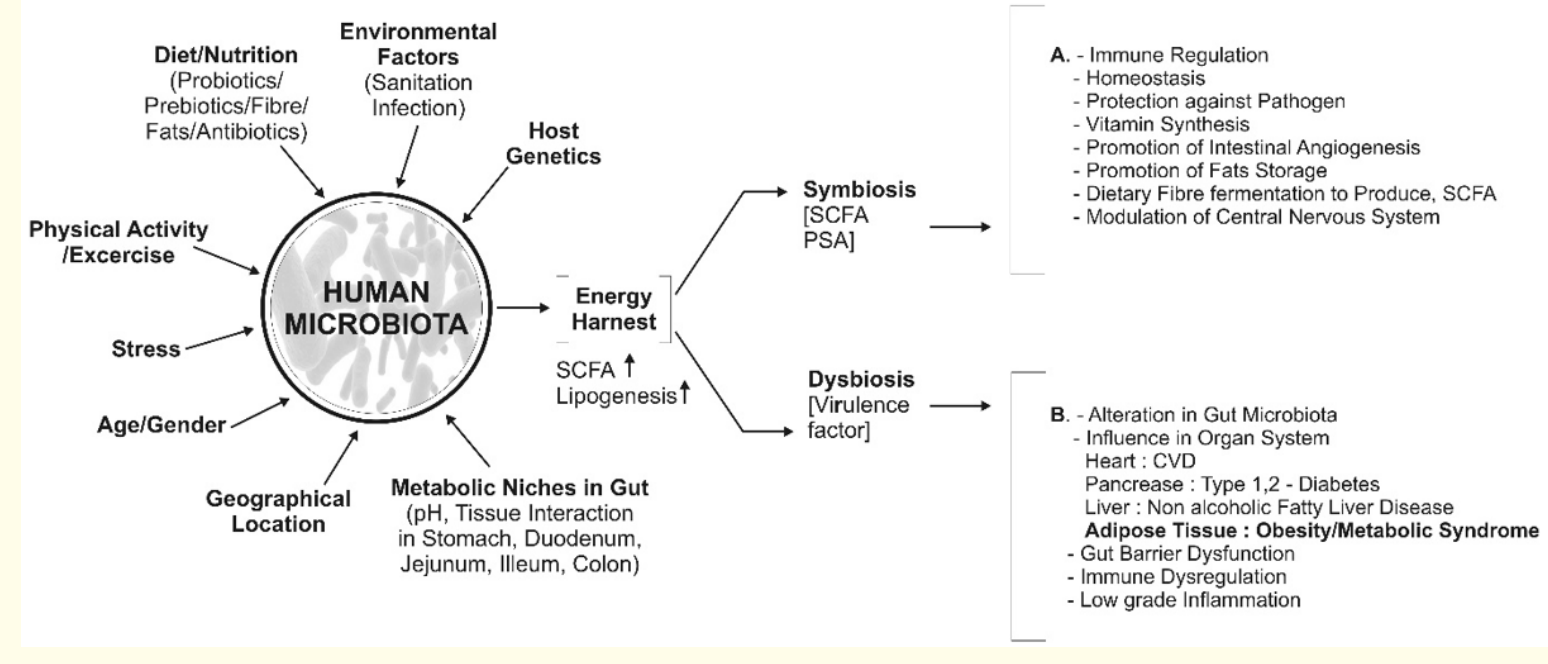

Figure 1: Factors affecting the composition and function of gut microbiota. While a healthy microbial community is vital to assist the host in maintaining good health, microbial dysbiosis can cause disease (Image created in CorelDraw).

Evidence from weight loss interventions reported induced diets influence gene richness in the low gene count (LGC) individuals leading to improved metabolic status [11] and indicates the link between long-term dietary habits and the structure of the gut microbiota [12]. Alterations in gut microbiota in severe obesity also showed decreased MGR (microbial gene richness) related to functional pathways linked with metabolic deteriorations [13]. These studies put forward a question that whether this low MGR is a cause or is it a consequence of obesity and of its duration. It represents a good biomarker of gut microbiota alteration, yet the issue concerning diet and gut microbiota in obesity needs more effective approach. Genetic factors contribute to the control of the physiologic response to caloric excess and therefore promote in the development and maintenance of obesity. Therefore, diet can aid in permanent adjustment of structure and functional feature in the microbiota.

Gut microbiota and functional aspects

The human body harbours a complex microbial population of both anaerobic and aerobic microbial communities called as gut microbiota that interact with each other and with their host, causing influence on the metabolic, immunologic and physiology and health of the host [14]. The genetic material of all microbes i.e. bacteria, fungi, protozoa and viruses inside the human body is the microbiome [15]. Human gastrointestinal tract has about 10 million genes which are associated with different microbial species and all genes have some beneficial impact on the health of the host (Table 1), with possible effects on the homeostasis of the immune system, conversion of food into useful nutrients and protection against pathogenic microorganisms invading [16,17]. Table 1 shows the composition of gut microbiota with different taxonomic variability in the gastrointestinal tract. Among the abundant bacterial domain Firmicutes (e.g. Clostridium and Bacillus spp) and gram positive Actinobacteria (e.g. Bifidobacterium spp) and gram negative Bacteroidetes (e.g. Bacteriodes spp and Prevotella) together account for more than $90 \%$, and in Archaea domain, a hydrogen-consuming methanogen viz, Methanobrevibacter sp. is present, influenced by number of gut environment factors such as $\mathrm{pH}$, oxygen, nutrients availability $[15,18]$.

Cox AJ, 2015 [18] very clearly classified the crucial roles of the resident microbiota in host functioning and subsequently in health and disease. Some of the major functions of the intestinal microbiota include:

1. Resistance to infection by pathogenic microorganisms through direct competition for nutrients and attachment sites, and production of antimicrobial substances;

2. Promotion of epithelial cell proliferation and differentiation to maintain an intact mucosal surface; 


\begin{tabular}{|c|c|c|c|c|c|}
\hline \multicolumn{2}{|r|}{ Phylum } & \multirow{2}{*}{$\begin{array}{l}\text { Prevalence } \\
\sim 50-65 \%\end{array}$} & \multirow{2}{*}{\begin{tabular}{|c} 
Different species \\
Clostridium, Bacillus sp., \\
Eubacteria, Roseburia, Lac- \\
tobacilli, L. fermentum
\end{tabular}} & \multirow{2}{*}{$\begin{array}{c}\text { Host Function } \\
\text { Ferment Fiber into butyrate, symbi- } \\
\text { onts to pathogens Butyrate produc- } \\
\text { tion. Role in cell proliferation. }\end{array}$} & \multirow{2}{*}{$\begin{array}{l}\text { Diet Sources } \\
\text { High fat diet + high } \\
\text { fiber }\end{array}$} \\
\hline $\begin{array}{l}\text { Major } \\
\text { Phyla }\end{array}$ & Firmicutes & & & & \\
\hline & Bacteroidetes & $\sim 25-42 \%$ & $\begin{array}{l}\text { Bacteroides, Prevotella, } \\
\text { Parabacteroides, Alistipes }\end{array}$ & $\begin{array}{l}\text { Polysaccharides degradation. Fer- } \\
\text { ment Fiber. }\end{array}$ & $\begin{array}{l}\text { Protein rich food, High } \\
\text { fiber diets especially } \\
\text { grains. }\end{array}$ \\
\hline & Actinobacteria & $\sim 3-13 \%$ & Bifidobacterium spp & Vitamin biosynthesis & Probiotics \\
\hline & Proteobacteria & $\sim 5-10 \%$ & E. coli & Role in GI Tract & \\
\hline \multirow[t]{6}{*}{$\begin{array}{l}\text { Minor } \\
\text { Phyla }\end{array}$} & Archaea & $<1 \%$ & $\begin{array}{l}\text { Methanobrevibacter, } \\
\text { Methanosphaera }\end{array}$ & Both convert hydrogen to methane. & \\
\hline & Deferribacteres & $<1 \%$ & & Degrade Iron & In GI Tract \\
\hline & Fusobacteria & $<1 \%$ & Fusobacterium nucleatum & $\begin{array}{l}\text { Proinflammatory colonic tumori- } \\
\text { genic factor }\end{array}$ & High meat diets \\
\hline & Melainabacteria & $<1 \%$ & & $\begin{array}{l}\text { Synthesize Vitamin B and K. Ferment } \\
\text { carbohydrate into ethanol, lactate } \\
\text { and formate. }\end{array}$ & $\begin{array}{l}\text { Plant based diets, } \\
\text { groundwater. }\end{array}$ \\
\hline & Streptococcus spp & $<2 \%$ & S. thermophilus & $\begin{array}{l}\text { Primary carbohydrate metabolism; } \\
\text { yielding lactic acid. }\end{array}$ & $\begin{array}{l}\text { Upper GI Tract; Milk } \\
\text { and dairy products, } \\
\text { eggs. }\end{array}$ \\
\hline & $\begin{array}{l}\text { Enterobacteria- } \\
\text { ceae }\end{array}$ & $<1 \%$ & $\begin{array}{l}\text { E. cloacae, E. coli, } \\
\text { Enterobacter, Citrobacter }\end{array}$ & $\begin{array}{l}\text { Aerobic cellular respiration; in GI } \\
\text { tract ferment sugars to various end } \\
\text { products. }\end{array}$ & Processed foods \\
\hline
\end{tabular}

Table 1: Overview of the composition of the human microbiome.

3. Promotion of the development of the gut-associated lymphoid tissue via initiation of dendritic cell maturation and differentiation of $\mathrm{B}$ and $\mathrm{T}$ lymphocytes; and

4. Energy harvest from non-digestible dietary starches and this function is very important in the context to obesity. Metagenomics studies discovered that increase in butyrate-producing Firmicutes and low abundance in Bacteroidetes species in distal microbial colony of obese patients and genetically obese mice, compared to their normal lean subjects [19] which indicates microbiota type and abundance also plays a role.

Efficient in nutrient digestion, gut microbiota ferments complex food components, synthesizes vitamins and other essential micronutrients, metabolizes dietary toxins and carcinogens, converts cholesterol and bile acids, assures the maturation of the immune system, affects the growth and differentiation of enterocytes, regulates intestinal angiogenesis, and protects against enteric pathogens [20]. Gut microbiota-host symbiotic relationship and intestinal homeostasis is usually maintained to offer benefits to human health until dysfunction of host machineries causing an imbalance called as dysbiosis, which may lead to pathogenesis and progression toward a broad spectrum of diseases [19] including immune disorders, susceptibility to infections and non-intestinal pathologies like cardiovascular diseases, obesity, diabetes, liver diseases [21]. Studies discussed gut microbiota dysbiosis probably promotes diet induced obesity and metabolic complications by a variety of mechanisms including immune dysregulation, altered energy regulation, altered gut hormone regulation and proinflammatory mechanisms [such as lipopolysaccharide endotoxins crossing the gut barrier and entering the portal circulation affecting the metabolic homeostasis and reproductive fitness in women [22] leading to polycystic ovary syndrome [PCOS] and insulin resistance development [23].

Animal Studies on gut microbiota established its role on host adiposity, a diet-lifestyle related condition. Body fats, accompanied by insulin resistance, adipocyte hypertrophy and increased levels of circulating leptin and glucose [24] and gut microbiota suppresses the intestinal expression of angiopoietin-like 4 (ANGPTL4), a circulating inhibitor of lipoprotein lipase (LPL) leading to increased hepatic lipogenesis in the host and suppress the skeletal muscle fatty acid oxidation through a metabolic pathway involving phosphorylation of AMP-activated protein kinase (AMPK) [25]. Reproductive conditions like infertility and PCOS and metabolic disorders in obesity are regulated via symbiotic interactions of the host energy metabolism and host immune system and gut microbiota [4,26]. Qi., et al. 2019, reported high prevalence of B. vulgatus, an anaerobic gram negative bacteria in the gut of women with PCOS and suggested presence of intestinal cytokines [Interleukin-22] that modulate immune response (production by T-helper lymphocytes) and influence bile acid metabolism which trigger $B$. vulgatus to contribute to PCOS pathology through GATA3 signalling pathways [23].

In colon, bacteria undergoes fermentation where non-digestible dietary polysaccharides [prebiotic] converted to monosaccharides 
to produce short-chain fatty acids (SCFAs) [mainly acetate, propionate, and butyrate] $[15,18]$. These SCFAs are the main energy source in colon epithelial cells and stimulate blood flow, gut hormone secretion, fluid and electrolyte intake, mucin release as well as gluconeogenesis and lipogenesis. Short-chain fatty acids could contribute to regulation of cell cycles, cell proliferation and apoptosis and are involved in regulation of intestinal permeability [18]. Gut microbiota in response to diet allows epithelial cells to express G-Protein Coupled Receptors which are free fatty acid receptor 2 and 3 (FFAR2 and FFAR3) that gets activated by acetate and butyrate [metabolites] respectively and aids in energy modulation. Increased production of SCFAs due to fiber rich diet influence eating behaviour. Similarly, increased action of gut peptides (PYY) and glucagon like peptides (GLP) decreases ghrelin action to have influence on appetite. Studies have also discussed how SCFA molecules act in inhibiting histone deacetylases (HDACs) or by acting as ligands for several G protein- coupled receptors GPRs; including GPR41 (also known as FFAR3), GPR43 (also known as FFAR2) and GPR109A (also known as HCAR2) and peroxisome proliferator- activated receptor- $\gamma$ (PPAR $\gamma$ ) [27-29].

\section{Diet - microbiome - host interactions}

Globalization has affected people's eating habits, leading to consume high-fat and high-calorie foods and different diets [traditional and modern] that shape the gut microbial community by supplying the nutrients and conditioning the intestinal microenvironment [30]. Diet may come as macronutrients, micronutrients, minerals, fats, bioactive nutrients and food additives interact with the gut microbiota to produce metabolites that modulate host metabolism in both beneficial and detrimental ways [29]. The interactions might be due to immuno-modulatory effects of the microbiota, downstream effects on host gene expression or alterations in the landscape of microbiota producing metabolites. The predominant effects of different dietary components on gut microbiota governing host physiology are depicted in the in table 2. Protein, fat and carbohydrates are the major components in diets of human results in metabolite production as end products due to of microbial-mediated degradation of protein and carbohydrates in the GI tract (GIT).

Fibres: Dietary fiber is an important food component derived from plants, which is present in very low concentration in processed foods compared to raw vegetables. Low fibre diet, fermentable fibre [inulin] affects Akkeransia, Bacteroides caccae and Bifidobacterium species and increase the production of Interleukin (IL)-22 or decrease production of mucus leading to risk of metabolic syndrome. Caecal and colonic microbiota ferments dietary fibre to produce SCFAs that serve as signalling molecules and hence influence metabolic pathways [31] and the remaining fibres are then excreted through feces [17]. Studies reported fibre rich food benefits in combating the metabolic syndrome, weight loss and insulin sensitivity improvement. Animal studies found barley- kernel based bread diet led to improved glucose tolerance and the subjects had high levels of Prevotella sp. that promoted hepatic glycogen storage in mice [32]. A very interesting fact came to limelight from a study that dietary regimens have cross generational consequences. For example, lack of dietary fibre reduced gut bacterial diversity in mice, which could be restored over a single generation. But if shortage in dietary fibre is over several generations, then it might result in permanent reduction of bacterial abundance to render extinction of important microbial taxa [33].

Fibres in wheat/bran, barley, oats, maize, rice have different impacts on gut microbiota. While wheat bran rich in dietary fiber, essential fatty acids with quantities of starch, protein, vitamins, and dietary minerals significantly influence microbial abundance and metabolites production on the other hand, rice grain, rich in fiber, minerals and vitamins and antioxidants, oils showed no significant change in bacterial abundance but induced increase in SCFA [34].

Carbohydrates: The digestibility of dietary carbohydrates depends on structural properties of and degree of polymerization [DP] and branching [35]. Indigestible carbohydrates termed as glycan [resistant starch, inulin, lignin, pectin, cellulose and fructo-oligosaccharides] derived from plant, animal, fungal and algal sources in the diet reach the large intestine is degraded by Bacteroides, Bifidobacterium and Ruminococcus genera, to produce glucose and ferment to form of acetate, propionate, formate, butyrate, lactate and succinate to initiates a complex cross-feeding metabolic network. Sulfate-reducing bacteria viz, methanogens and acetogens consume fermented products such as hydrogen gas in human gut [29]. Complex carbohydrates promotes health affect the diversity and microbial abundance evident from a study that reported polymer of fructose molecules [i.e. $\beta 2 \rightarrow 1$-fructan] has a direct modulatory effects on immunity, which in turn affects the colonizing microbiota [36]. Supplemental disaccharides, oligosaccharides, and polysaccharides of various doses increases Bacteroides abundance. Supplementation of Maltitol [Disaccharide] positively affect Bifidobacterium, Bacteroides, Lactobacilli, Eubacteria. Fermented soybean milk (containing raffinose/stachytose), Oligosaccharide positively affect Bifidobacterium and Lactobacilli but negatively affect Clostridia, Collinsella and Streptococcus. Resistant starch in Polysaccharide group affect positively Actinobacteria (Bifidobacterium) but negatively affect Firmicutes (Ruminococcaceae). The change in microbial functionality and abundance affects cardiometabolic and inflammatory process that are related to obesity and type 2 diabetes [35].

Prebiotics such as oligosaccharides, conjugated linoleic acid and milk sphingomyelin have been suggested to enhance metabolism $[37,38]$, as it exert bifidogenic effect, providing a fermentable food source, allowing increase in the growth of specific beneficial microbial populations such as Lactobacilli and Bifidobacteria influencing physical, biochemical, and metabolic parameters related to obesity [39]. 


\begin{tabular}{|c|c|c|c|}
\hline $\begin{array}{l}\text { Nutritional/Dietary } \\
\text { components }\end{array}$ & Influence on microbiota & Influence on host health & Reference \\
\hline Whole Grain Diet & $\begin{array}{l}\text { Relative abundance of Enterobac- } \\
\text { teriaceae decreased; Butyrate-pro- } \\
\text { ducing Lachnospira and Roseburia } \\
\text { increased. }\end{array}$ & $\begin{array}{l}\text { Energy balance regulation, Plasma alkyl- } \\
\text { resorcinols increased and maintained body } \\
\text { weight. }\end{array}$ & Karl JP., et al. [57] \\
\hline Low Carbohydrate Intake & $\begin{array}{l}\text { Decreases Eubacterium rectale, Bifi- } \\
\text { dobacterium and Roseburia species }\end{array}$ & Unclear. & Murphy EF., et al. [16] \\
\hline $\begin{array}{l}\text { High Carbohydrate diet } \\
+ \text { High-fat +High-protein } \\
\text { diets }\end{array}$ & $\begin{array}{c}\text { Promote development of Bacteroide- } \\
\text { tes; Favors Prevotella genus. }\end{array}$ & $\begin{array}{l}\text { Ferment polysaccharides and indigestible } \\
\text { carbohydrates. Benefits the gut by SCFAs } \\
\text { production. }\end{array}$ & $\begin{array}{l}\text { G.D. Wu., et al. [53]; Ra- } \\
\text { joka MSR., et al. [17] }\end{array}$ \\
\hline High protein diet & $\begin{array}{c}\text { Causes riched Bacteroides associated } \\
\text { enterotype. Reduce Firmicutes. }\end{array}$ & Decrease in weight & $\begin{array}{l}\text { David La., et al. [52]; G.D. } \\
\text { Wu., et al. [53] }\end{array}$ \\
\hline $\begin{array}{l}\text { High Fat Diet and High } \\
\text { Calorie/ Western Diet }\end{array}$ & $\begin{array}{c}\text { Progressive increase in Firmicutes } \\
\text { [Mollicutes sp] and Reductions in } \\
\text { Bacteroidetes. No relation to markers } \\
\text { of energy harvest. }\end{array}$ & $\begin{array}{l}\text { Obesity leading to decrease in the gut mi- } \\
\text { crobiota diversity. Metabolic Pathways }\end{array}$ & $\begin{array}{l}\text { Murphy E F., et al. [16]; } \\
\text { Turnbaugh PJ., et al. } \\
\text { [54]; Shen W., et al. [55] }\end{array}$ \\
\hline $\begin{array}{l}\text { Less vegetable, fish and } \\
\text { fruits }\end{array}$ & Reduced microbiome & $\begin{array}{c}\text { Enhance of inflammation, triglyceride level, } \\
\text { insulin resistance and low density lipopro- } \\
\text { teins cholesterol }\end{array}$ & Matijasic BB., et al. [56] \\
\hline Gluten Free Diet & $\begin{array}{c}\text { Beneficial bacteria decreased, while } \\
\text { unhealthy bacteria increased. Reduc- } \\
\text { tions in polysaccharides intake. }\end{array}$ & $\begin{array}{l}\text { Exerted lower production of cytokines and } \\
\text { chemokines [TNF } \alpha \text {, IFN } \gamma \text { and IL-8] and } \\
\text { anti-inflammatory cytokines [IL-10] on } \\
\text { peripheral blood mononuclear cells pro- } \\
\text { inflammatory [PBMCs]. i.e. exerted lower } \\
\text { immune stimulatory effects. }\end{array}$ & Sanz, Y [58] \\
\hline $\begin{array}{l}\text { High Fiber Diet/ Plant } \\
\text { based Fibers }\end{array}$ & $\begin{array}{l}\text { Influence microbial colonization. } \\
\text { Enhance mucus and anti-microbial } \\
\text { peptide production. Major driver of } \\
\text { Prevotella-type microbiota. }\end{array}$ & $\begin{array}{c}\text { Fermentation variables results in resulted } \\
\text { in a greater short-chain fatty acid [SCFA] } \\
\text { concentration [e.g. acetic and butyric acids], } \\
\text { which help in regulating host metabolism, } \\
\text { immune system, and cell proliferation }\end{array}$ & $\begin{array}{l}\text { Makki, K., et al. [60]; } \\
\text { Nakayama J., et al. [30] }\end{array}$ \\
\hline $\begin{array}{l}\text { Calorie restricted diet } \\
{[\mathrm{CR}] \text { enriched with fibre }} \\
\text { and protein }\end{array}$ & $\begin{array}{c}\text { Associated with A. muciniphila } \\
\text { abundance helped in } \\
\text { anti-inflammatory effects. }\end{array}$ & $\begin{array}{c}\text { Improvement in insulin sensitivity markers } \\
\text { and other clinical parameters in over- } \\
\text { weight/obese adults. }\end{array}$ & Dao MC., et al. [59] \\
\hline
\end{tabular}

Table 2: Studies on influence of dietary in take and its practices on gut-microbiota.

\section{Fats}

High intake of dietary fat is associated with cardiovascular diseases (CVDs) and obesity but with some exceptions considering quantity and the source of fat. Fats have differential effects on the host and induced changes in the gut microbiome [40]. Saturated fat modifies the microbiome to promote metabolic syndrome, colitis or central nervous system autoimmunity, altering the immune landscape in the gut and such detrimental effects that can be partially inherited. High fat or high sugar diet increases Firmicutes, Mollicutes and Eubacterium population to increase lactate, acetate and butyrate and at the same time decreases Bacteroidetes to increase metabolic syndrome [29]. High-fat diet (HFD) or Western diet [high-fat, high-sugar] is associated with a decrease in Bacteroidetes levels and an increase in Firmicutes (Table 2) leading to composition change triggering range of functional effects such as increased sucrose metabolism, urea metabolism, membrane transport systems, metabolism of cofactors and vitamins and protein folding, sorting and degradation [29,41].
Studies on low-fat high-fiber diet, and a high-fat high-protein modern diet reported considerable difference in gut microbiota at a phylum level of Firmicutes-to-Bacteroidetes (F/B) ratio. Fat intake correlated positively with this ratio and negatively with the relative abundance of the family Prevotellaceae/genus Prevotella [30] in obesity. The enterotype of gut microbiome are associated with [high-fat high-protein] diets like modern artificial foods with a high content of animal fat and protein and (low-fat high-carbohydrate) diets like traditional low-fat diets with a high content of plant polysaccharides or meat-free vegetarian food $[42,43]$.

Iron

Diet rich in iron is long known to help in preventing and treating anemia but on other hand it is found to result in dysbiosis and bloom of pathogens [44]. Iron is absorbed predominantly in the duodenum, prebiotic fermentation can decrease $\mathrm{pH}$ in the colon, promoting the reduction of Fe (III) to Fe (II) and thus favouring iron absorption [45]. Through fermentation, probiotic bacteria that use 
prebiotic fibers [carbon source] to generate large amounts of SCFAs (lactate, pyruvate, and acetate) molecules and these are used by colon bacteria to produce propionate and butyrate. Butyrate in colon is considered important for its anti-inflammatory properties, promotion of the expansion of regulatory T cells via the inhibition of histone deacetylation and induction of IgA production by mucosal B cells $[39,44,45]$.

\section{Other dietary components}

Non-caloric artificial sweeteners contains saccharin, aspartame affects microbial metabolites results in considerable undesirable effects on host health. High sugar diets contribute to metabolic disorders. The role of non-nutritive sweeteners in metabolic syndrome takes place through three potential mechanisms which are: 1) NNS interacting with sweet taste receptors, 2) NNS interfering with gut microbiota composition, and 3) NNS interfering with learned responses to sweetness. The dietary sugar upregulates hepatic uptake and metabolism of fructose, which leads to liver lipid accumulation, dyslipidemia, decreased insulin sensitivity, and increased uric acid levels leading to metabolic risk factors such as abdominal obesity, high triglyceride level, low HDL cholesterol level, high blood pressure, and high fasting blood sugar [46].

Diet containing red and processed meat, rich in L-carnitine are metabolized by the gut microbiota to trimethylamine [TMA], transported by the portal circulation to the liver and converted into trimethylamine $\mathrm{N}$ - oxide [TMAO] by flavin mono-oxygenases [47]. This is associated with an increased risk of developing hyperlipidaemia and hypercholesterolaemia and CVD. Red meat is also rich in haem, which is reported to link with colonic cytotoxicity and hyper-proliferation [48]. Haem-rich diet leads to a bloom of mucin- degrading bacteria such as A. muciniphila, leading to impaired intestinal barrier function due to degradation of the mucous layer. Again, Lactobacillus species in gut can bind to heterocyclic amines produces by processed meat and are reported to have protection of the host from DNA damage and neoplasia [49].

Processed foods contains emulsifiers can erode the host's protective epithelial mucous layer and lead to dysbiosis-mediated lowgrade inflammation and the promotion of the metabolic syndrome. Emulsifiers such as carboxymethyl cellulose and polysorbate-80 are reported to induce low grade inflammation and obesity and promotes colitis in mice. Broad use of emulsifying agents might contribute to an increased societal incidence of obesity/metabolic syndrome and other chronic inflammatory diseases promoting detrimental effects [50]. Again, vegetable and fruit juice diets decreased the abundance of faecal Firmicutes and Proteobacteria but increased Bacteroidetes and Cyanobacteria, inducing beneficial functional changes in metabolism [51].

\section{Challenges and Future Considerations}

The present scenario of gut microbiota research in relation to diet intervention is in exploratory stage. Germ-free animal's stud- ies has allowed discovery of the significant role of gut microbiota in host metabolism but the challenge remains in successful translational results from animal experiments to clinical applications which is yet to be studied. More research is needed on functional genes of gut microbiota combined with multi-omics research through correlative analysis of multidimensional data which sounds very challenging but it will be very effective in identifying species related to disease at the strain level.

Till date, animal experimentation including mouse models using germ-free technology coupled with functional genomics approaches, has defined relative contributions of microbiota and host factors and diet in the GI tract. Despite the importance of microbiology and nutrigenomics field, the components of the human microbiota still remain poorly understood. This may require in depth knowledge on molecular mechanisms of microbiota and its metabolic potential towards human physiology. The host genetic expressions in response to microflora should be explored further to identify novel targets as biomarkers for nutrient processing and utilization.

Earlier studies have limitations on number of participants and the short term interventions. Limitations due to lack of in-depth biological and biochemical information has held back to address the complex physiological link among diets, microbes, host, and other underlying factors. Studies have reported correlation between obesity and enterotype (Prevotellaceae or Bacteroidaceae species) is linked to either fats type or carbohydrate type diets. But it is not clearly understood whether the altered gut microbiota is a cause of obesity or just a consequence of altered dietary habits or the process behind effects.

Screening of dietary fibers, prebiotics or food can define efficient strategies to replenish gut microbiome with essential missing microbes. A good understanding of impact of dietary nutrition on gut microbiota associated with mechanism underlying the human health conditions such as host glycemic control, inflammation is an absolute necessity. Also, diet-microbiota interactions will help to develop a personalized nutrition approach that would target and individualize gut microbiota manipulation to combat metabolic diseases and improve overall host metabolic health.

The microbiota composition also changes with different features of fat intake rather than genetics in obesity. The possibility of microbial adaptation to diet and time should be considered in future studies. Emphasis on changes in the proportions of Firmicutes and Bacteroidetes species in obesity can be helpful for further insights.

Further studies on the nutritional quality of the different types of diet viz, Gluten free, Mediterranean, Plant based diet etc. and its effects on gut ecology and health in larger population group and over longer periods are needed. Reviewing the studies indicates 
that altered gut microbiota may be a sign of a modern diet-associated obesity and it can be treated with proper and effective management.

\section{Conclusion}

The gut microbiome represents an interesting platform for research as it significantly contribute to the rapid increase in obesity rates. It connects host and environmental factors and act as a modulator of energy homeostasis and fat deposition. The multiple parameters of host such as sociodemographics, location, environmental exposures, genetics and medical conditions or treatments, encompassing with diet components has differential influence on composition and functional features, metabolites, growth dynamics of microbiota. This complex interactions together make up leading to low grade inflammation, hyperlipidaemia, hypertension, glucose intolerance and diabetes, and result in obesity and its metabolic complications in the host.

These associations have been studied extensively including short- to long-term dietary effects and from local to global structural changes in the gut microbial community. Disturbance in the interaction between nutrition, metabolism and microbiome may constitute an important factor in the deregulation of normal host homeostasis. The compelling link calls for a good understanding of the mechanisms involved in the interactions between diet composition, gut microbiota and obesity. Diet is a major environmental factor influencing gut microbiota diversity and functionality and evidence suggests that gut microbiota diversity and metabolic function play an important role in the development of obesity and related metabolic disorders. Obesity is frequently thought as the outcome of a relative imbalance in energy intake versus energy expenditure. Enhanced energy harvest from dietary intake, due to an alteration in microbial composition, has been highlighted as a potential contributor to the pathogenesis of obesity as diet through gut microbiota can have effects on development of the immune system, nutrient absorption, tissue generation, morphogenesis, and bone homeostasis metabolism. Diets rich in fats, western diets or low fiber diet disrupt gut microbiota barrier function, which might be improved by fibre supplementations.

Most studies show an imbalance characterized by a lower diversity in microbial species in overweight and obese people, indicating role in body weight management, energy metabolism through the diet, in regulation of fat storage, lipogenesis and fatty acid oxidation and therefore are key to many aspects of human health and thus influence the development and progression of obesity. Studies like gene approach, genome-wide linkage studies and genome-wide association claimed to make obesity a genetic disorder to understand pathogenesis of obesity and related metabolic diseases but gut microbiota with diet/food habits and lifestyle changes equally holds importance as it greatly and tremendously contributes to the current obesity trends. The functional role of the gut microbiome in humans cannot be ignored, as it has become a growing important component to understand the complete picture of obesity. The picture seems contrasting when research on obesity or body weight management, as a field has advanced now because of significant progress in nutrigenomics, metabolomics including RNA silencing, epigenetics, and different areas that may bring about the rise of new technologies and techniques to understand the association between microbiota and obesity.

The therapeutic effects of diet including probiotics and prebiotics on body weight, fat deposition, lipid profile, and chronic inflammation state has now become a mandatory investigating points. Studies from different perspectives have been made to understand the multifactorial pathophysiology of obesity. The understanding of host-microbiome interaction can assist in devising dietary interventions, which can be used for prophylactic or treatment purposes in a variety of medical conditions including obesity. Multidisciplinary research in this field will be helpful to provide therapeutic potential of gut microbes in metabolic disorders and obesity treatment. Therefore, manipulation of the microbiota has become a potential therapeutic tool now, which is a rapidly advancing field in microbiome research. More clinical studies that can be translated into clinical practice to provide a greater understanding of the host-microbiome interactions that has impact on Obesity.

\section{Acknowledgement}

We extend our gratitude and appreciation to Mr. Ashish Debnath, who contributed in creating the image in CorelDraw.

\section{Bibliography}

1. World Health Organization: "Obesity and overweight" (2018).

2. Voreades Noah., et al. "Diet and the development of the human intestinal microbiome". Frontiers in Microbiology (2014): 5.494 .

3. Singh Rasnik K., et al. "Influence of diet on the gut microbiome and implications for human health". Journal of Translational Medicine 15.1 (2017): 73.

4. Legro Richard S. "Obesity and PCOS: implications for diagnosis and treatment". Seminars in Reproductive Medicine 30.6 (2012): 496-506.

5. McPherson Ruth. "Genetic contributors to obesity". The Canadian Journal of Cardiology 23 (2007): 23A-27A.

6. World Health Organization. “Global Health Observatory (GHO) data" (2016).

7. Bray GA and Ryan DH. "Clinical evaluation of the overweight patient". Endocrinology 13 (2000): 167-186.

8. Monteiro CA., et al. "Ultra-processed products are becoming dominant in the global food system". Obesity Reviews 14.2 (2013): 21-28.

9. Al-Assal K., et al. "Gut microbiota and obesity". Clinical Nutrition Experimental 20 (2018): 60-64.

10. Vermeulen E., et al. "A combined high-sugar and high-saturated-fat dietary pattern is associated with more depressive symptoms in a multi-ethnic population: The HELIUS (Healthy Life in an Urban Setting) study". Public Health Nutrition 20 (2017): 2374 - 2382. 
11. Cotillard A., et al. "Dietary intervention impact on gut microbial gene richness". Nature 500 (2013): 585-588.

12. Karlsson F., et al. "Gut metagenome in European women with normal, impaired and diabetic glucose control”. Nature 498 (2013): 99-103.

13. Rothschild D., et al. "Environment dominates over host genetics in shaping human gut microbiota". Nature 555 (2018): 210-215.

14. O'Hara Ann M and Fergus Shanahan. "The gut flora as a forgotten organ”. EMBO Reports 7.7 (2006): 688-693.

15. Gerard P. "Gut microbiota and obesity". Cellular and Molecular Life Sciences 73 (2016): 147-162.

16. Murphy EF., et al. "Composition and energy harvesting capacity of the gut microbiota: relationship to diet, obesity and time in mouse models". Gut 59.12 (2010): 1635-1642.

17. Riaz Rajoka MS., et al. "Interaction between diet composition and gut microbiota and its impact on gastrointestinal tract health". Food Science and Human Wellness 6.3 (2017): 121130.

18. Cox AJ., et al. "Obesity, inflammation, and the gut microbiota". The Lancet Diabetes and Endocrinology 3.3 (2015): 207-215.

19. Kho ZY and Lal SK. "The Human Gut Microbiome - A Potential Controller of Wellness and Disease". Frontiers in Microbiology 9 (2018): 1835.

20. Hooper LV., et al. "How host-microbial interactions shape the nutrient environment of the mammalian intestine". Annual Review of Nutrition 22 (2002): 283-307.

21. Backhed F., et al. "Defining a healthy human gut microbiome: current concepts, future directions, and clinical applications". Cell Host Microbe 12.5 (2012): 611-622.

22. Silva MSB and Giacobini P. "Don't Trust Your Gut: When Gut Microbiota Disrupt Fertility". Cell Metabolism 30.4 (2019): 616-618.

23. Qi X., et al. "Gut microbiota-bile acid-interleukin-22 axis orchestra polycystic ovary syndrome". Nature Medicine 25 (2019): 1225-1233.

24. Bäckhed Fredrik., et al. "The gut microbiota as an environmental factor that regulates fat storage". Proceedings of the National Academy of Sciences of the United States of America 101.44 (2004): 15718-15723.

25. Backhed F., et al. "Mechanisms underlying the resistance to diet-induced obesity in germ-free mice". Proceedings of the National Academy of Sciences of the United States of America 104.3 (2007): 979-984.
26. Boulangé CL., et al. "Impact of the gut microbiota on inflammation, obesity, and metabolic disease". Genome Medicine 8 (2016): 42 .

27. Cani PD., et al. "Gut microbiota fermentation of prebiotics increases satietogenic and incretin gut peptide production with consequences for appetite sensation and glucose response after a meal". The American Journal of Clinical Nutrition 90.5 (2009): 1236-1243.

28. Tarini J and Wolever TM. "The fermentable fibre inulin increases postprandial serum short-chain fatty acids and reduces free-fatty acids and ghrelin in healthy subjects". Applied Physiology, Nutrition, and Metabolism (2010). 35.1 (2010): 9-16.

29. Niv Zmora Jotham Suez and Eran Elinav. "You are what you eat: diet, health and the gut microbiota". Nature Reviews Gastroenterology and Hepatology 16.1 (2019): 35-56.

30. Nakayama J., et al. "Impact of Westernized Diet on Gut Microbiota in Children on Leyte Island" Frontiers in Microbiology 8 (2017): 197.

31. Koh A., et al. "From dietary fiber to host physiology: shortchain fatty acids as key bacterial metabolites" Cell 165 (2016): 1332-1345.

32. Kovatcheva-Datchary P., et al. "Dietary fiber-induced improvement in glucose metabolism is associated with increased abundance of Prevotella". Cell Metabolism 22 (2015): 971-982.

33. Sonnenburg ED., et al. "Diet- induced extinctions in the gut microbiota compound over generations". Nature 529 (2016): $212-215$

34. Jefferson A and Adolphus K. "The Effects of Intact Cereal Grain Fibers, Including Wheat Bran on the Gut Microbiota Composition of Healthy Adults: A Systematic Review". Frontiers in $\mathrm{Nu}$ trition 6 (2019): 33.

35. Vinke PC., et al. "The Role of Supplemental Complex Dietary Carbohydrates and Gut Microbiota in Promoting Cardiometabolic and Immunological Health in Obesity: Lessons from Healthy Non-Obese Individuals". Frontiers in Nutrition 4 (2017): 34 .

36. Fransen F., et al. “ $32>1$-fructans modulate the immune system in vivo by direct interaction with the mucosa in a microbiota-independent fashion". Frontiers in Immunology 8 (2017): 154.

37. Cani PD. et al. "Selective increases of bifidobacteria in gut microflora improve high- fat-diet- induced diabetes in mice through a mechanism associated with endotoxaemia". Diabetologia 50 (2007): 2374-2383. 
38. Norris GH., et al. "Milk sphingomyelin improves lipid metabolism and alters gut microbiota in high fat diet- fed mice". Journal of Nutritional Biochemistry 30 (2016): 93-101.

39. Cerdó T., et al. "The Role of Probiotics and Prebiotics in the Prevention and Treatment of Obesity". Nutrients 11.3 (2019): 635.

40. Siri- Tarino PW., et al. "Meta- analysis of prospective cohort studies evaluating the association of saturated fat with cardiovascular disease". The American Journal of Clinical Nutrition 91 (2010): 535-546.

41. Wu Meng., et al. "Genetic determinants of in vivo fitness and diet responsiveness in multiple human gut Bacteroides". Science 350.6256 (2015): 5992.

42. Zhang J., et al. "Mongolians core gut microbiota and its correlation with seasonal dietary changes". Scientific Reports 4 (2014): 5001.

43. Ruengsomwong S., et al. "Microbial community of healthy Thai vegetarians and non-vegetarians, their core gut microbiota and pathogens risk". Journal of Microbiology and Biotechnology 26 (2016): 1723-1735.

44. Dostal Alexandra., et al. "Iron depletion and repletion with ferrous sulfate or electrolytic iron modifies the composition and metabolic activity of the gut microbiota in rats". The Journal of Nutrition 142.2 (2012): 271-277.

45. Yeung CK., et al. "Prebiotics and iron bioavailability-Is there a connection?" Journal of Food Science 70 (2005): R88-R92.

46. Liauchonak I., et al. "Non-Nutritive Sweeteners and Their Implications on the Development of Metabolic Syndrome". Nutrients 11.3 (2019): 644.

47. Koeth Robert A., et al. "Intestinal microbiota metabolism of Lcarnitine, a nutrient in red meat, promotes atherosclerosis". Nature Medicine 19.5 (2013): 576-585.

48. Ijssennagger N., et al. "Gut microbiota facilitates dietary heme- induced epithelial hyperproliferation by opening the mucus barrier in colon". Proceedings of the National Academy of Sciences of the United States of America 112 (2015): 1003810043.

49. Zsivkovits M., et al. "Prevention of heterocyclic amine-induced DNA damage in colon and liver of rats by different lactobacillus strains". Carcinogenesis 24 (2003): 1913-1918.

50. Chassaing B., et al. "Dietary emulsifiers impact the mouse gut microbiota promoting colitis and metabolic syndrome". Nature 519.7541 (2015): 92-96.
51. Henning Susanne M et al. "Health benefit of vegetable/fruit juice-based diet: Role of microbiome". Scientific Reports (2017): 7.

52. David Lawrence A., et al. "Diet rapidly and reproducibly alters the human gut microbiome”. Nature 505.7484 (2014): 559563.

53. GD Wu., et al. "Linking long-term dietary patterns with gut microbial enterotypes". Science 334 (2011): 105-108.

54. Turnbaugh Peter J., et al. "Diet-induced obesity is linked to marked but reversible alterations in the mouse distal gut microbiome". Cell Host and Microbe 3.4 (2008): 213-223.

55. W Shen., et al. "Influence of dietary fat on intestinal microbes inflammation, barrier function and metabolic outcomes". Journal of Nutritional Biochemistry 25 (2014): 270-280.

56. Matijašić BB., et al. "Association of dietary type with fecal microbiota in vegetarians and omnivores in Slovenia". European Journal of Nutrition 53 (2014): 1051-1064.

57. Karl J Philip., et al. "Substituting whole grains for refined grains in a 6-wk randomized trial favorably affects energy-balance metrics in healthy men and postmenopausal women". The American Journal of Clinical Nutrition 105.3 (2017): 589-599.

58. Sanz Yolanda. "Effects of a gluten-free diet on gut microbiota and immune function in healthy adult humans". Gut Microbes 1.3 (2010): 135-137.

59. Dao MC., et al. "Akkermansia muciniphila and improved metabolic health during a dietary intervention in obesity: Relationship with gut microbiome richness and ecology". Gut 65 (2016): 426-436.

60. Makki K., et al. "The Impact of Dietary Fiber on Gut Microbiota in Host Health and Disease". Cell Host and Microbe 23.6 (2018): 705-715.

\section{Assets from publication with us}

- Prompt Acknowledgement after receiving the article

- Thorough Double blinded peer review

- Rapid Publication

- Issue of Publication Certificate

- High visibility of your Published work

Website: https://www.actascientific.com/

Submit Article: https://www.actascientific.com/submission.php Email us: editor@actascientific.com

Contact us: +919182824667 